\title{
Cytology of Some Members of the Family Iridaceae
}

\author{
Arun Kumar Sharma and Archana Sharma \\ Cytogenetics Laboratory, Botany Department, Calcutta University, \\ Calcutta 19 , India
}

Received November 16,1960

\section{Introduction}

Detailed cytological works have been done on several members of the family Iridaceae (Mather 1932, Randolph 1934, 1947, Simonet 1934, 1951, Randolph and Randolph 1954, Mitra 1955, etc.). However, in some genera, the chromosome morphology and its bearing on their affinities have not yet been thoroughly studied. Recently, in Cipura paludosa, the cytology of which had not been examined in detail earlier, structural hybridity and the existence of several biotypes differing in chromosome numbers, have been recorded (Sharma and Talukdar 1959).

A cursory examination by the author of several other genera, such as Babiana, Tritonia, Tigridia and Moraea reveals the existence of intraspecific polyploidy in some of them, unobserved so far. Aneuploid individuals are also found to occur. Such cases of aneuploidy and polyploidy are not uncommon in other genera of Iridaceae. It was therefore thought worthwhile to work out the cytology of the aneuploid and polyploid types of different species of these genera. A simultaneous thorough study of the chromosome morphology, so far unknown, of these different species and their aneuploid and polyploid derivatives is necessary in order to trace the relationships of the different taxonomic units. In the present paper, this aspect of the work has been carried out with the help of recently improved methods for karyotype study.

\section{Material and methods}

In the present study, seven different varieties, distributed under four genera of the family Iridaceae, have been included, namely,

1. Babiana sulphurea Ker-Gawl.

2. Tritonia crocata Ker-Gawl.

3. T. crocata Ker-Gawl., Type I.

4. Tigridia pavonia Ker-Gawl.

5. Moraea iridioides Linn. var. Mcleyii.

6. M. iridioides Linn. var. bicolor, and

7. M. iridioides Linn. var. johnsonii.

The plants were obtained from the Chandra Nursery at Rhenock in Sikkim, grown at an altitude of nearly 5000 feet under temperate conditions. 
Tritonia crocata Type I was supplied by the Nursery as Montbretia crocata. But as it is a synonym of $T$. crocata, it has been dealt with in the text as $T$. crocata. This differs from the normal $T$. crocata only in having narrower and smaller leaves. The identification of all species was confirmed from the Indian Botanical Gardens herbarium at Sibpore.

The bulbs were grown in a mixture of loose soil and sawdust in flower pots in the gardens of the Calcutta University College of Science. They were kept in shade.

Healthy root-tips were cut out and treated in different pretreatment chemicals, like aesculine, coumarin, p-dichlorobenzene, oxyquinoline, etc. (Tjio and Levan 1950, Sharma and Bal 1953, Sharma and Sarkar 1955, Sharma and Mookerjea 1955). Best results were obtained by treating in saturated aesculine solution at $12-16^{\circ} \mathrm{C}$ for one to one and half hours. The root-tips were then heated in a mixture of $2 \%$ aceto-orcein solution and normal hydrochloric acid $(9: 1)$ for a few seconds and finally squashed in $1 \%$ aceto-orcein solution. For making the preparations permanent, the slide containing the smear was inverted in $n$-butyl alcohol. After the coverglass had been detached, it was freshly mounted on the slide with euparal.

Drawings were made in a Zeiss microscope with an apochromatic objective N.A. 1.3 and an eyepiece of $\times 12$ at a table magnification of $\times 1800$ approximately. In the diagrams, the chromosomes with secondary constrictions have been drawn in outline. The figures have been reduced to half size in the reproduction.

\section{Observations}

The somatic chromosome numbers of the different varieties, so far studied, have been found to range from $2 n=14$ to $2 n=60$. Nuclei with varying number of chromosomes, either lower or higher than the normal one, are observed within the same tissue in most species. The variations include both structural and numerical alterations of chromosomes. The number occurring in the highest frequency is taken as the normal number for the species.

\section{Genus-Babiana}

Only one species was investigated.

\section{B. sulphurea Ker-Gawl $2 \mathrm{n}=14$}

Fourteen chromosomes are observed in the normal somatic complement. They are, on an average, medium-sized, as compared with the other members of Iridaceae studied. Size difference is very distinct, ranging from $2.1 \mu$ to $5.6 \mu$. Six chromosomes bear secondary constrictions.

A detailed analysis of the karyotype shows the presence of the follow. ing types of chromosomes (Figs. 1 and 1a):

Type A-One pair of comparatively long chromosomes, each with two 
constrictions, primary and secondary, located at nearly submedian to nearly subterminal positions at the two opposite ends of the chromosome. The middle segment is much longer than the side ones.
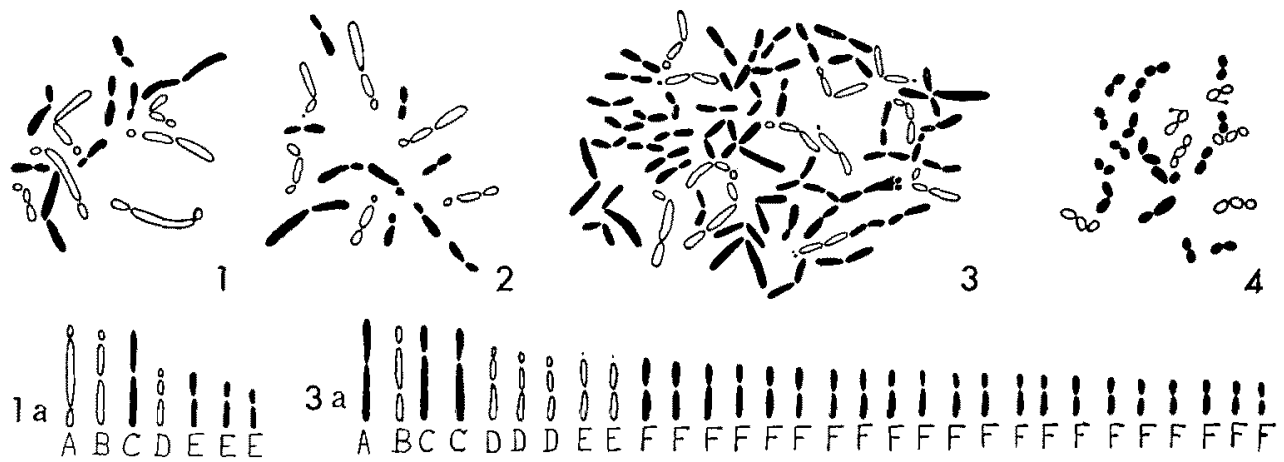
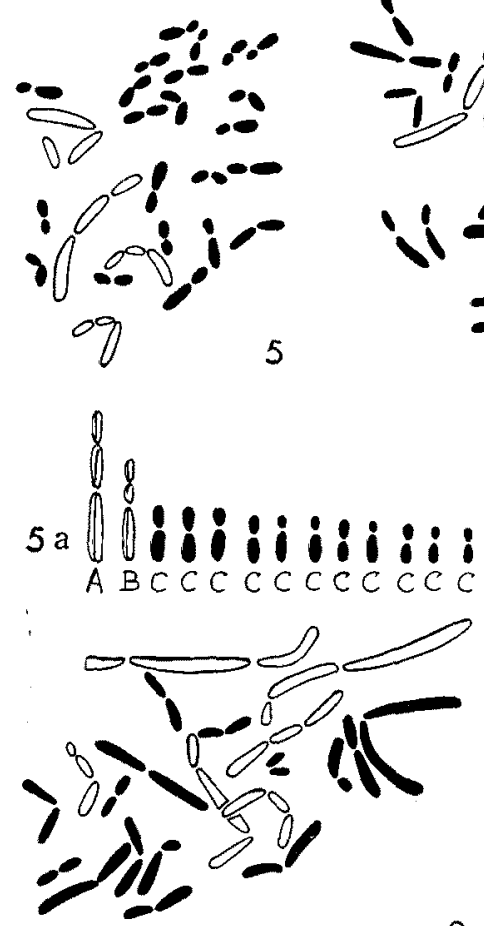
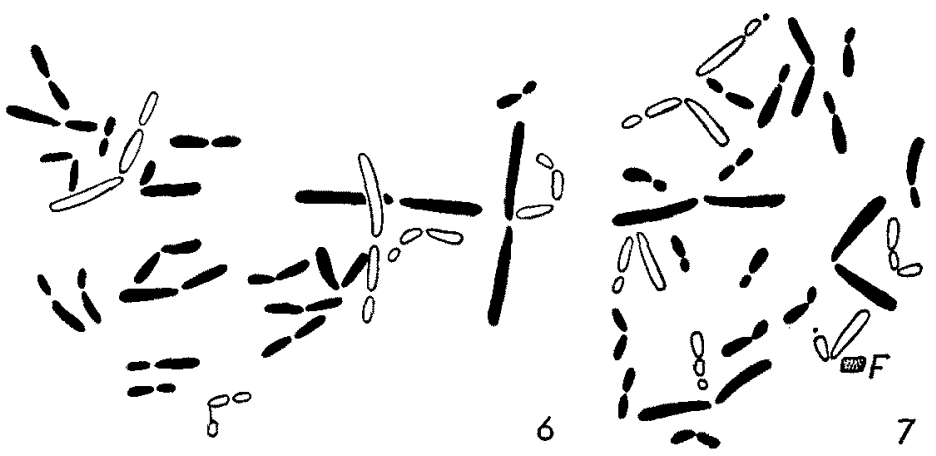
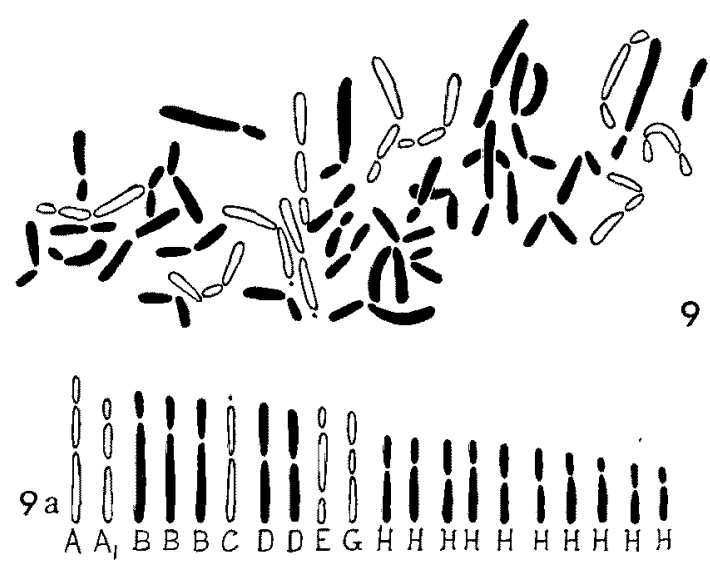

Figs. 1-9. 1, la and 2, Babiana sulphurea $2 \mathrm{n}=14$. Normal somatic metaphase, idiogram and variation nucleus with 16 chromosomes respectively. 3 and $3 a$, Tritonia crocata $2 n=60$. Normal somatic metaphase and idiogram respectively. 4, T. crocata Type I $2 \mathrm{n}=22$. Normal somatic metaphase. 5, 5a,6,7 and 8, Tigridia pavonia $2 \mathrm{n}=26$. Normal somatic meta. phase, idiogram and variation nuclei with $24,24+1 \mathrm{~F}$ and 21 chromosomes respectively. 9 and $9 \mathrm{a}$, Moraea iridioides var. Mcleyii $2 \mathrm{n}=40$. Normal somatic metaphase and idiogram respectively.

Type B-One pair of long chromosomes each with two constrictions, primary and secondary, one nearly median in position and the other sub- 
terminal at the distal end of the shorter arm.

Type C-A pair of long chromosomes with median primary constrictions.

Type D-A pair of nearly short chromosomes, each with two constrictions, primary and secondary, one nearly median and the other nearly subterminal at the distal end of the shorter arm.

Type E-Three pairs of nearly short to short chromosomes, with median primary constrictions.

A variation nucleus has been observed with sixteen chromosomes. In it, in addition to three altered $\mathrm{A}$, two $\mathrm{B}$, one $\mathrm{C}$ and four $\mathrm{E}$ chromosomes, five chromosomes with submedian primary constrictions and one new chromosome with a nearly submedian primary constriction and a satellite at the distal end of the short arm, are also present (Fig. 2).

Genus-Tritonia

$T$. crocata and another variety were studied.

2. $T$. crocata Ker-Gawl. $2 \mathrm{n}=60$

The normal somatic nuclei of this species contain sixty chromosomes. Size difference is marked, ranging from $1.7 \mu$ to $6 \mu$. The chromosomes form a graded series from long to short. Twelve of them bear secondary constrictions.

On the basis of their morphology and size, the chromosomes can be divided into the following types (Figs. 3 and 3 a):

Type A-A pair of long chromosomes with median primary constrictions.

Type B-A pair of long chromosomes, each with two constrictions, primary and secondary, located at submedian and nearly submedian positions at the opposite ends of the chromosomes.

Type C-Two pairs of long chromosomes, shorter than A, with nearly submedian primary constrictions.

Type D-Three pairs of medium-sized chromosomes, each with two constrictions, primary and secondary, one median in position and the other nearly submedian to submedian at the distal end of one of the arms.

Type E-Two pairs of medium-sized chromosomes, each with a median primary constriction and a small satellite at the distal end of one of the arms.

Type F--Twenty-one pairs of chromosomes, grading from medium sized to short, with nearly median to median primary constrictions.

3. T. crocata. Type I $2 \mathrm{n}=22$

Twenty-two chromosomes are seen in the somatic nuclei. They are much shorter than those of $T$. crocata. Size difference ranges from $1.4 \mu$ to $2.5 \mu$. This variety differs from $T$. crocata both in chromosome number and karyotype. Four chromosomes bear secondary constrictions (Fig. 4).

As compared with $T$. crocata, the complement of this variety consists of one pair of $A$, one pair of $B$, one pair of $C$, one pair of $D$ and seven pairs of $F$ type of chromosomes. All the types are much reduced in size. Genus-Tigridia

Only one species was worked out. 
4. Tigridia pavonia Ker. Gawl. $2 \mathrm{n}=26$

The normal somatic complement of this species contains twenty-six chromosomes. They show marked and abrupt difference in size, from long
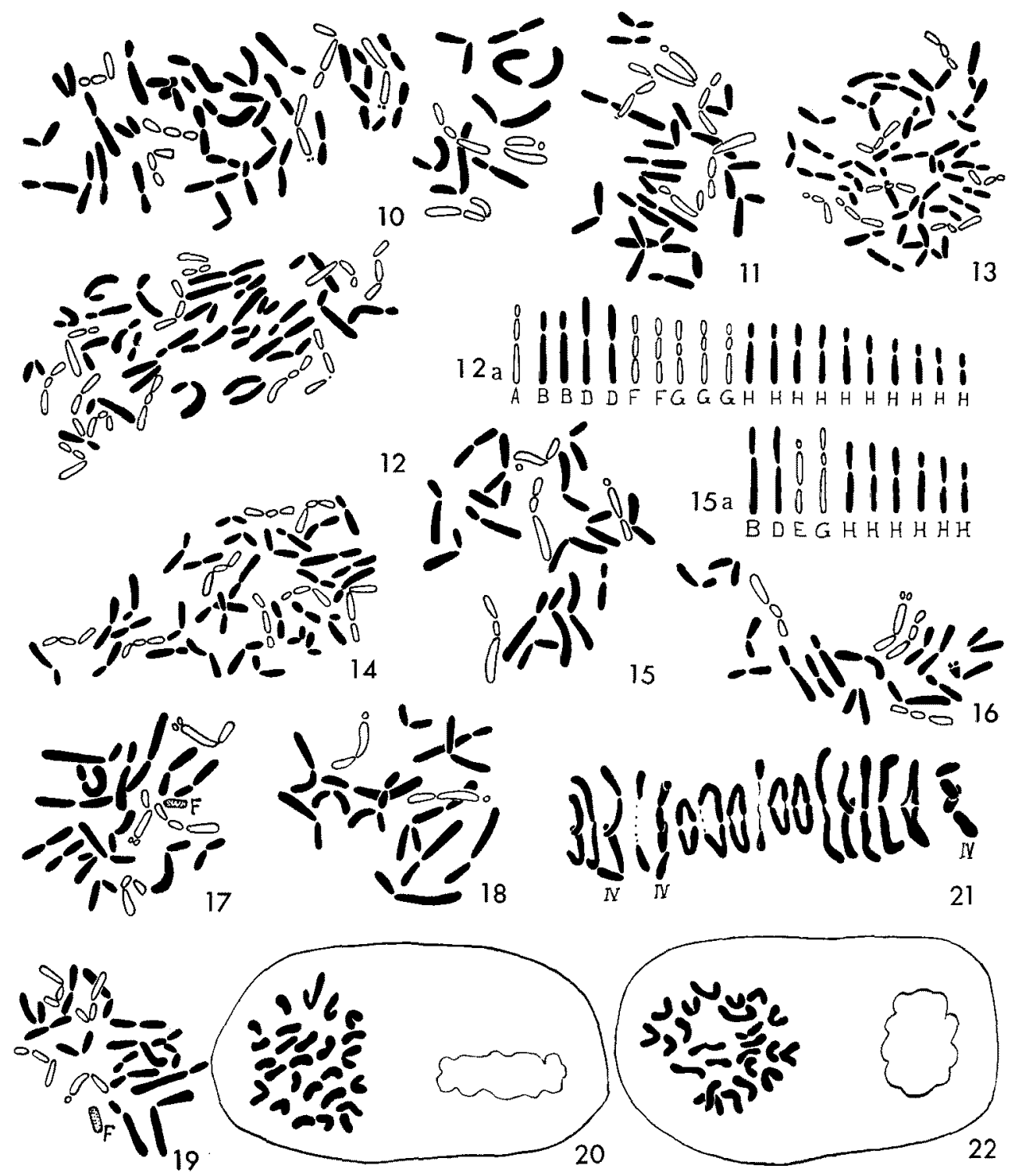

Figs. 10-22. 10 and 11, Moraea iridioides var. Mcleyii. Variation nuclei with 35 and 32 chromosomes respectively. $12,12 \mathrm{a}, 13$ and $14, M$. iridioides var. bicolor $2 \mathrm{n}=40$. Normal somatic metaphase, idiogram and variation nuclei with 41 and 38 chromosomes respectively. $15,15 \mathrm{a}, 16,17,18$ and 19, M. iridioides var. johnsonii $2 \mathrm{n}=20$. Normal somatic metaphase, idiogram and variation nuclei with $19,19+1 \mathrm{~F}, 16$ and $18+1 \mathrm{~F}$ chromosomes respectively. 20-22, M. iridioides var. Mcleyii. Meiotic stages (vide text).

to short. The size range varies between $2.1 \mu$ to $8.8 \%$. Four chromosomes bear secondary constrictions (Figs. 5 and $5 \mathrm{a}$ ). 
A detailed analysis of the karyotype shows the presence of the following distinct chromosomal types:-

Type A-One pair of long chromosomes, each having two constrictions, primary and secondary, one median and the other nearly submedian in position.

Type B-One pair of medium-sized chromosomes, each with two constrictions, primary and secondary, one median and the other nearly submedian in position, lying close to each other on one of the arms.

Type C-Eleven pairs of nearly short to short chromosomes with median to nearly submedian primary constrictions.

In addition to the normal set, variation nuclei with $24,24+1 \mathrm{~F}$ and 21 chromosomes have also been recorded. The plate with twenty-four chromosomes (Fig. 6) differs from the normal one in having two $\mathrm{A}$ and seventeen $\mathrm{C}$ chromosomes and also two new long chromosomes with median primary constrictions (S) and three new medium-sized chromosomes with both primary and secondary constrictions $(\mathrm{T})$.

The nucleus with twenty-four chromosomes and one fragment contains two altered $\mathrm{A}$, one $\mathrm{B}$, fourteen $\mathrm{C}$ chromosomes and four new (S) and one new (T) chromosomes. A new pair with submedian primary constriction and a satellite at the distal end of the short arm, is also seen (Fig. 7).

Fig. 8 shows a plate with twenty-one chromosomes. It possesses one $\mathrm{A}$, two $\mathrm{B}$, thirteen $\mathrm{C}$, two $(\mathrm{S})$ and one $(\mathrm{T})$ chromosomes and also a new pair with primary and secondary constrictions located at opposite ends of the chromosomes.

Genus-Moraea

Three varieties, belonging to one species $M$. iridioides, were studied. The chromosomes are, on an average, long, as compared with the other members of Iridaceae studied. The chromosome numbers are forty in two and twenty in one variety, indicating the presence of a polyploid series. The chromosomes of the different varieties resemble each other in their gross morphology.

On the basis of their detailed morphology and size, the following general types of chromosomes can be recognized:

Type A-Long chromosome with two constrictions, primary and secondary, one median and the other nearly submedian in position.

Type B-Long chromosome with submedian primary constrictions.

Type C-Long chromosome with a median primary constriction and a satellite at the distal end of one of the arms.

Type D-Long chromosome with median primary constriction.

Type E-Long chromosome with two constrictions, primary and secondary, at submedian to nearly submedian positions at opposite ends of the chromosome. The middle segment is much longer than the side ones.

Type F-Long chromosome with two constrictions, primary and secon- 
dary, one nearly submedian in position and the other located in the middle of the longer arm.

Type G-Long chromosome with two constrictions, primary and secondary, one median and the other submedian to nearly submedian in position.

Type $\mathrm{H}-$ Chromosomes with median to submedian primary constrictions, grading from nearly long to nearly short.

The karyotypes of the different varieties differ from each other in having different combinations of these types.

5. M. iridioides Linn. var. Mcleyii $2 \mathrm{n}=40$

Forty chromosomes have been observed in the normal somatic nuclei. Size difference is present, ranging from $3.1 \mu$ to $8.4 \mu$. Ten chromosomes bear secondary constrictions (Figs. 9 and $9 a$ ).

The normal complement consists of, two pairs of $A$, three pairs of $B$, one pair of $C$, two pairs of $D$, one pair of $E$, one pair of $G$ and ten pairs of $\mathrm{H}$ type of chromosomes. One $\mathrm{A}$ pair is shorter than the other $\mathrm{A}, \mathrm{B}$ and $C$ pairs. The primary constrictions of $B$ pairs grade from submedian to nearly submedian in position. The two constrictions of $G$ pair are rather close to each other. The $\mathrm{H}$ chromosomes range from medium-sized to nearly short.

Variation nuclei bearing thirty-five and thirty-two chromosomes have been seen. Fig. 10 shows a metaphase with thirty-five chromosomes. Two C, one $\mathrm{E}$ and three $\mathrm{G}$ chromosomes are present, in addition to $\mathrm{H}$ type chromosomes.

Fig. 11 shows thirty-two chromosomes in the set. Four $\mathrm{B}$, one $\mathrm{C}$, two $\mathrm{D}$, one $\mathrm{E}$, two $\mathrm{F}$, three $\mathrm{G}$ and the rest $\mathrm{H}$ chromosomes are observed. A new chromosome with primary and secondary constrictions, at nearly submedian and subterminal positions, is also seen.

In meiotic metaphase $\mathrm{I}$, fourteen bivalents and three quadrivalents are observed (Fig. 21). Irregular numbers, like twenty-six and twenty-four, are seen in second division metaphase stages (Figs. 20 and 22).

\section{M. iridioides Linn. var. bicolor $2 \mathrm{n}=40$}

The normal somatic metaphase contains forty chromosomes. They are, on an average, medium-sized and form a graded series. Size difference ranges between $2.5 \mu$ and $6.7 \mu$. Twelve chromosomes bear secondary constrictions (Figs. 12 and 12a).

A detailed analysis of the normal karyotype shows one pair of $A$, two pairs of $B$, two pairs of $D$, two pairs of $F$, three pairs of $G$ and ten pairs of $\mathrm{H}$ type of chromosomes. The $\mathrm{A}$ pair is short as compared to the previous variety. One $D$ pair is slightly longer than the other and both of them are longer than the $A$ and $B$ types. In one $G$ pair, the two constrictions lie rather close to each other. The $\mathrm{H}$ chromosomes grade from comparatively long to short.

Variation nuclei have been recorded with forty-one and thirty-eight 
chromosomes. Fig. 13 shows a metaphase with forty-one chromosomes. Four altered $A$, two $B$, two $D$, three $G$ and thirty $H$ chromosomes are present in it.

Fig. 14 shows a nucleus bearing thirty-eight chromosomes. Of them, two are of $\mathrm{A}$, two $\mathrm{F}$, four $\mathrm{G}$ and the remaining of $\mathrm{H}$ type.

7. $M$. iridioides Linn. var. johnsonii. $2 \mathrm{n}=20$

Twenty chromosomes are observed in the normal somatic complement. They grade in size from long to nearly short. Size range varies between $3.5 \mu$ and $6.7 \mu$. Four chromosomes bear secondary constrictions (Figs. 15 and 15a).

The normal set contains one pair of $B$, one pair of $D$, one pair of $E$, one pair of $G$ and six pairs of $H$ type of chromosomes. In the $E$ pair, one constriction is nearly submedian and the other subterminal at the distal end of the longer arm. In the $G$ pair, the two constrictions lie very close to each other and the chromosomes are longer than the B, D and E ones. The $\mathrm{H}$ pairs form a grading series. Variation nuclei bearing $19,19+1 \mathrm{~F}, 16$ and $18+1 \mathrm{~F}$ chromosomes are also on record.

In the metaphase with nineteen chromosomes (Fig. 16), one $\mathrm{E}$ and three $\mathrm{G}$ chromosomes are present in addition to $\mathrm{H}$.

Fig. 17 shows nineteen chromosomes and one fragment in the set, of which two are of $B$, two $D$, two $E$, two $G$ and the remaining of $H$ type.

In the variation nucleus with sixteen chromosomes, two $\mathrm{B}$, two $\mathrm{D}$, two $\mathrm{E}$ and ten $\mathrm{H}$ type members are seen (Fig. 18).

Fig. 19 shows a metaphase plate with eighteen chromosomes and one fragment. One $A$, two $B$, two $D$, one $E$, two $G$ and the rest $H$ type chromosomes are present.

\section{Discussion}

In Babiana stricta, the only species of this genus studied so far, discrepancies exist in the reports of the chromosome number (vide Darlington and Wylie 1955). Brittingham (1934) observed twelve chromosomes in the somatic cells of this species while Sugiura (1931) reported fourteen chromosomes in the individuals studied by him. These varying reports and the fact that chromosomal biotypes occur in different genera of Iridaceae, as men. tioned at the beginning of this paper, show that this species too exhibits such variation at an intraspecific level. This phenomenon may have a profound significance in evolution. The diploid chromosome number of $B$. sulphurea has been found to be fourteen, evidently drawing it near B. stricta in taxonomic position. The occurrence of this number in both species may indicate that $n=7$ is deep-seated for this genus. In that case, the individual of $B$. stricta with twelve chromosomes may have been derived from those with fourteen chromosomes, through the elimination of one chromosome from the gametic set. However, several more species of this genus should be studied to confirm the exact basic number. As $B$. sulphurea shows three 
pairs of long and four pairs of comparatively shorter chromosomes in its complement, it appears that this genus represents a distinct line of evolution and is not closely related to the other genera studied here.

The correct position of Babiuna in this family, with relation to the other genera, must be determined from a thorough comparison of the karyotypes of its members with those of other genera.

The karyotype of Tigridia pavonia shows an abrupt size difference amongst the chromosomes. Of the two pairs of chromosomes with secondary constrictions, one is very long and the other slightly shorter. The remaining twenty-two chromosomes form a homogeneous series, being short and possessing the constrictions at almost the same position. This abrupt size difference, indicating asymmetry in the karyotype, denotes the advanced state of this species. In Babiana sulphurea, size difference among the chromosomes is distinct, but not so marked as in Tigridia pavonia. The latter genus also shows intraspecific aneuploidy, because Sakai (1952) recorded individuals with twenty-eight chromosomes while Brittingham (1.c.) and the present author observed plants with twenty-six chromosomes. Without further investigation, it is difficult to state as to which number represents the basic one for this genus. The extreme size difference of chromosomes in $T$. pavonia, observed here, suggests a distinct line of evolution for this genus, separate from the other genera studied by the present author. This karyotype, if found to be characteristic for this genus, may have arisen by crossing two different species, having long and short chromosomes respectively. Karyotypes with such size difference have been seen in Cipura paludosa as well, where also the chromosome number varies from twelve to fourteen.

In Tritonia crocata and $T$. crocata type $I$, the diploid numbers have been observed to be sixty and twenty-two respectively. In the same species, collected from South Africa, Brittingham (l.c.) observed twenty chromosomes in the somatic set, indicating that the East Himalayan individuals of $T$. crocata, studied by the present author, are high polyploids. Evidently, the Himalayan forms are derivatives of the original diploid type, representative individuals of which have been found in South Africa.

The chromosome number of $T$. crocata type $I$ is unlike that of any other individual of $T$. crocata. It is significant that in the allied genus Crocosmia (Brittingham l.c., Muerman and Suomalainen 1946), the same chromosome number has been found. Further, the species of this genus cross readily with species of Tritomia. Several species of Crocosmia, like those of Montbretia too, have been transfered under Tritonia. This variety of T. crocata -Type I,-as mentioned before, was supplied by the Nursery as Montbretia crocata, which is now a synonym of Tritonia crocata as the genus Montbretia is now merged with Tritonia (vide Index Kewensis upto 1953). The karyotype of $T$. crocata is markedly dissimilar to that of $T$. crocata Type $\mathrm{I}$, as 
the former has much longer chromosomes than the latter. The morphology, as shown by the idiograms, also varies widely. It therefore appears that $T$. crocata Type I would be in a more natural systematic position if included under a separate genus, Montbretia, or under the allied genus Crocosmia, provided that the taxonomic characters and other aspects of study will support it.

The varieties of Moraea iridioides studied here resemble Tigridia pavonia in several aspects. In Moraea, the chromosome numbers are multiples of ten, except in $M$. edulis (Sakai, l.c.). Two of the three varieties of $M$. iridioides studied show forty chromosomes while one shows twenty. Their karyotypic differences suggest the occurrence of profuse structural alterations of chromosomes during their evolution. All of them show a graded karyotype, as observed in Tritonia crocata as well. On the basis of similarities in chromosome number, size, morphology and even to some extent external characters, it can be assumed that these two genera represent diversities of a common line of evolution.

Another remarkable phenomenon, noted during the course of the present investigation in several species, is the occurrence of variation in number and structure of the chromosomes in the somatic tissue, along with the normal complement. Such inconstancy in the chromosome complements, noted in a few somatic cells, taken in conjunction with the existence of a large number of aneuploid individuals of the different species, suggests that such somatic variations may play a significant role in their speciation, either directly through vegetative means, or by their persistence in the germ cells (Sharma 1956).

\section{Summary}

Cytological study has been carried out on seven species of Iridaceae, distributed under four genera, namely,

1. Babiana sulphurea $2 \mathrm{n}=14$

2. Tritonia crocata $2 \mathrm{n}=60$

3. $T$. crocata Type $\mathrm{I} 2 \mathrm{n}=22$

4. Tigridia pavonia $2 \mathrm{n}=26$

5. Moraea iridioides var. Mcleyii $2 \mathrm{n}=40$

6. $M$. iridioides var. bicolor $2=40$

7. M. iridioides var. johnsonii $2 \mathrm{n}=20$

On the basis of the data obtained and taking into account previous reports, it has been suggested that $n=7$ is probably the basic number for Babiana. A distinct line of evolution has been drawn for this genus, unrelated with the three other genera studied here.

Tigridia pavonia shows abrupt size difference among the members of its complement and a different line of evolution for this genus too has been proposed. 
Tritonia crocata Type I, formerly included under Montbretia, has been observed to be cytologically allied to the genus Crocosmia. Its inclusion under the latter or its status as a member of a separate genus Montbretia, is urged.

Tritonia crocata and the three varieties of Moraea iridioides show affinities in chromosome number and karyotype. A common evolutionary line had been assumed for them.

Numerical and structural alterations of chromosomes in the somatic tissue have been noticed and their significance in evolution has been emphasized.

\section{References}

Brittingham, W. H. 1934. Cytological studies on some genera of the Iridaceae. Amer. J. Bot. 21: $77-82$.

Darlington, C. D. and Wylie, A. 1955. Chromosome atlas of flowering plants. George Allen and Unwin Ltd., London.

Index Kewensis 1893-1953. Vols. 1 and 2 and supplements I-XI, Clarendon Press, Oxford. Mather, K. 1932. Chromosome variation in Crocus. J. Genet. 26: 129.

Meurman, O. and Suomalainen, E. 1946. Ann. Ak. Sci. Fenn. 4: 11. (quoted from Darlington and Wylie 1955).

Mitra, J. 1955. Karyotype analysis of bearded Iris. Ph. D. thesis, Cornell Univ., Ithaca, N. Y.

Randolph, I. F. 1934. Chromosome numbers in native American and introduced species and cultivated varieties of Iris. Bull. Amer. Iris Soc. 52: 61-66.

- 1947. Chromosomes and classification of bearded Iris. Bull. Amer. Iris Soc. 108: 68-78.

- and Randolph, F. R. 1954. Chromosome number in Iris. Bull. Amer. Iris Soc. 133: 15-18.

Sakai, B. 1952. Zytologische Untersuchungen bei Iridaceen I. Cytologia 17: 104-111.

Sharma, A. K. 1956. A new concept of a means of speciation in vegetatively reproducing plants. Caryologia 9: 93-130.

- and Bal, A. K. 1953. Coumarin in chromosome analysis. Stain Techn. 28: 255-257.

- and Mookerjea, A. 1955 Paradichlorobenzene and other chemicals in chromosome work. Stain. Techn, 30: 1-7.

- and Sarkar, S. K. 1955. A new technique for the study of chromosomes in Palms. Nature 176: 261.

- and Talukdar, C, 1959. Cytotaxonomical studies on some members of the Iridaceae with special reference to the structural heterozygosity of Cipura paludosa Aubl. The Nucleus 1: $63-84$.

Simonet, M. 1934. Nouvelles recherches cytologiques et genetiques chez les Iris. Ann. Sci. Nat. Bot. $10_{\mathrm{e}}$ Ser. 16: 229-283.

- 1951. Sur les meiose de quelques hybrides d'Iris. 1. Hybrides diploides et triploides Pogoniris grands. Compt. Rend. Acad. Sci. (Paris) 232: 650-652.

Sugiura, T. 1931. A list of chromosome number in Angiospermous plants. Bot. Mag. Tokyo 45: 353-355.

Tjio, J. H. and Levan, A. 1950. The use of oxyquinoline in chromosome analysis. Anal Estac. Exp. de Aula-dei 2: 21-64. 